\title{
Relationship Between Non-Invasive Blood Pressure (NIBP), on First Day of Life with Birth Weight
}

\author{
Chawla $\mathrm{G}^{1}$, Maini $\mathrm{B}^{2}$, Bhardwaj $\mathrm{AK}^{3}$
}

\begin{abstract}
Introduction: India faces lot of neonatal morbidity. Non-invasive blood pressure (NIBP) monitoring is being used for monitoring sick new borns as a safe and cost effective method of blood pressure monitoring. But there is no available information on normal values of NIBP for neonates in India. With more stress of government (and advancement) in new born care, establishing normative data of NIBP in Indian newborns should be high priority. Material and Methods: This was an observational cohort study done in a level III neonatal intensive care unit. 164 clinically stable babies on day 1 of life were included. Blood pressure was measured by the Oscillometric method. Results: Progressive increase of blood pressure was seen with increasing birth weight. $10^{\text {th }}, 50^{\text {th }}, 75^{\text {th }}, 95^{\text {th }}$ Centile curves were drawn from available data. For very low birth weight babies (< 1500grams), the values of systolic, diastolic and mean BP $( \pm 2$ standard deviations (S.D.)) were found to be $53.7 \pm 1.7,29.1 \pm 1.7$ and 41 $\pm 1.1 \mathrm{~mm} \mathrm{Hg}$ respectively. For low birth weight babies, i.e. with birth weight 1500-2499 grams, the values for systolic, diastolic and mean $\mathrm{BP}( \pm 2$ S.D. $)$ were $62.2 \pm 2.5,34.8 \pm 2.4$ and $46.6 \pm 2.4$ $\mathrm{mm} \mathrm{Hg}$ respectively. For normal weight babies (more than 2499 grams), the systolic, diastolic and mean BP ( \pm 2 S.D.) were $72.8 \pm$ $4.2,43 \pm 4$ and $55.3 \pm 4 \mathrm{~mm} \mathrm{Hg}$ respectively. The rise in systolic blood pressure with rising birth weight was highly significant $(p<0.001)$. Similar were the results for diastolic and mean BPs, ( $p<0.001$ for both), Conclusion: Blood pressure on day 1 of life appears to be directly related to birth weight. The effect is seen in all (i.e. systolic, diastolic and mean) blood pressure values.
\end{abstract}

\section{Introduction}

mprovements in neonatal care have resulted in increasing survival of sick newborns ${ }^{1}$. In newborn, circulatory system undergoes greatest change in physiology. These differences in circulatory system also reflect in arterial blood pressure of neonate. The newborn is incapable of maintaining adequate cerebral blood flow by arteriolar vasodilatation when perfusion pressure falls and is prone to cerebral ischemia with only modest fall in blood pressure ${ }^{2}$. The signs and symptoms in neonates with abnormal blood pressure may be absent or non specific, thereby making blood pressure monitoring in neonates mandatory ${ }^{3}$.
'Garima Chawla, Post doctoral fellow in Paediatric endocrinology (SGRH New Delhi), 'Baljeet Maini, Associate Professor, Department of Paediatrics, Maharishi Markandeshwar Institute of Science and Research (MMIMSR), Mullana, Ambala, India. ${ }^{3}$ Anand Kumar Bhardwaj, Professor, Department of Paediatrics, Maharishi Markandeshwar Institute of Science and Research (MMIMSR), Mullana, Ambala, India

\author{
Address for correspondence: \\ Dr. Baljeet Maini \\ Associate Professor, Department of Paediatrics \\ MMIMSR, Mullana \\ Ambala, India \\ E-mail:mainibaljeet@gmail.com \\ Tel: +91 8059931611
}

\section{How to cite}

Chawla G, Maini B, Bhardwaj AK. Relationship Between Non-Invasive Blood Pressure (NIBP), on First Day of Life with Birth Weight. J Nepal Paediatr Soc 2015;35(2):141-146.

doi: http://dx.doi.org/10.3126/jnps.v35i2.12911

This work is licensed under a Creative Commons Attribution 3.0 License.

\section{(c) (i)}

Invasive procedures like intra arterial catheters provides accurate and reproducible blood pressure measurements But this method is associated with many hazardous complications ${ }^{4}$. Recent advancements include development of noninvasive methods like oscillometric method 
and doppler ultrasound probe. Of these oscillometric method is most often adopted in neonatal intensive care units because of its extensive advantages ${ }^{5}$.

There is no available information, in currently available literature, on blood pressure values in Indian newborns. Establishment of normal values of blood pressure in newborn is thus necessary so that pathological alterations can be detected especially in sick neonates. We therefore conceptualized this study in our centre to add to literature and hoping, for better clinical care of Indian neonates, in future.

\section{Material and Methods}

This observational cohort study was conducted in tertiary level Neonatal Unit of Maharishi Markandeshwar Institute of Medical Science and Research, (MMIMSR) Hospital, Ambala, India. Purposive method of sampling was used to recruit the babies with the aim of including as much as number of eligible babies in the study duration i.e. between November 2010 and July 2012. Parents were informed about the study and a written consent was taken. Prior approval of the Institution Ethics Committee was taken for conducting the study.

Babies were categorised as extremely low birth weight $(<1000$ grams), very low birth weight (10001499 grams), low birth weight (1500 grams to 2499 grams) as per internationally accepted criteria ${ }^{1}$.

Exclusion criteria: Following babies were excluded from the study group:

- $\quad$ Extremely low birth weight $(<1000$ gram birth weight), owing to unit protocol of administering iv fluids soon after birth.

- Newborn babies admitted in nursery for any other sickness

- Babies with congenital malformations requiring immediate intervention

- Newborns on following drugs

a Corticosteroids

․ Theophylline

口 Vasopressor drugs

- Maternal history of diabetes, substance abuse

The study cohort was subjected to non-invasive blood pressure (NIBP) measurement by oscillometric method between 6 to 24 hours of birth. Cuff bladders of different sizes were chosen in a way so as to have a width covering $2 / 3$ rd of upper arm and encompassing the entire arm. Before recording the blood pressure it was ensured that the baby was in calm and quiet position. Babies were left undisturbed for at least 15 minutes after applying the cuff or until the baby slept or was in a quiet awake state, in absence of struggling, crying and movement. No sedation was given to any baby. The blood pressure was recorded in the right arm using multipara monitor (Excello Eco, BPL health care, Pallakad, India) by oscillometric method. Three readings of blood pressure were taken at two minute interval with the child lying in supine position and in a restful state. Values were discarded if there was considerable movement of the infant. Averages of the three systolic, mean and diastolic readings were used for analysis.

Statistical analysis: Data was analysed over an SPSS version 15.5 for WINDOWS-7.Pearson Chi Square test was used to obtain ' $p$ ' value for correlation between BP and birth weight; and BP and period of gestation at the time of birth. (' $p$ ' value of $<0.05$ being significant).

\section{Results}

One hundred and sixty four newborns were included in the study. There were $87(53.1 \%)$ males and $77(46.9 \%)$ female babies. Seventeen (10.4\%) were early preterm, 46 (28\%) late preterm and 101 (61.6\%) term newborns. Out of 164 newborns, 96 (58.5\%) were $\geq 2500$ grams; 59 (36\%) were low birth weight and $9(5.5 \%)$ were very low birth weight. None of the subjects included were <1000grams. The newborns included in our study had gestational age of 33-41 weeks with mean gestation age of $37 \pm 2$ weeks and a mean birth weight of $2505 \pm 585$ grams. Figure 1 shows systolic, diastolic and mean blood pressures measured by oscillometric method in newborns included in our study against their birth weights. It is evident from figure 1 that there is a progressive rise in systolic, diastolic and mean arterial blood pressure values with an increase in birth weight. Further, we derived normogram of 10th, 50th, 75th and 95th percentiles for systolic, diastolic and mean BPs for birth weight from available data (Figures 2,3 \&4). For babies less than 1500grams, the values of systolic, diastolic and mean BP were found to be $53.7 \pm 1.7,29.1 \pm 1.7$ and 41 $\pm 1.1 \mathrm{~mm} \mathrm{Hg}$ respectively.

For low birth weight babies, i.e. with birth weight 1500-2499 grams, the values for systolic, diastolic and mean BP were $62.2 \pm 2.5,34.8 \pm 2.4$ and $46.6 \pm 2.4 \mathrm{~mm}$ $\mathrm{Hg}$ respectively.

For babies more than 2499 grams, the systolic, diastolic and mean BP were $72.8 \pm 4.2,43 \pm 4$ and 55.3 4 $4 \mathrm{~mm} \mathrm{Hg}$ respectively. 
The rise in systolic blood pressure with rising birth weight was highly significant $(\chi 2=296.255, \mathrm{df}=4, p=$ $0.00)$. Similar were the results for diastolic and mean BPs, $(\chi 2=249.009, \mathrm{df}=4, p=0.00)$ and $(\chi 2=265.476$, $\mathrm{df}=4, p=0.00$ ) respectively (Tables $1,2,3$ ).

Table 1: Systolic BP (SBP) and Birth weight

\begin{tabular}{|c|c|c|c|c|}
\hline $\begin{array}{c}\text { Birth weight } \\
\text { (Grams) }\end{array}$ & \multicolumn{3}{|c|}{ No; of new borns with SBP } & Total \\
\hline & $\leq 56$ & $56.1-66.2$ & $\geq 66.3$ & \\
\hline $1001-1499$ & 9 & 0 & 0 & 9 \\
\hline $1500-2499$ & 1 & 59 & 2 & 62 \\
\hline$\geq 2500$ & 0 & 1 & 92 & 93 \\
\hline TOTAL & $\mathbf{1 0}$ & $\mathbf{6 0}$ & $\mathbf{9 4}$ & $\mathbf{1 6 4}$ \\
\hline
\end{tabular}

Table 2: Diastolic blood pressure and Birth weight

\begin{tabular}{|c|c|c|c|c|}
\hline \multirow{2}{*}{$\begin{array}{c}\text { Birth weight } \\
\text { (Grams) }\end{array}$} & \multicolumn{3}{|c|}{$\begin{array}{c}\text { No; of new borns with DBP } \\
\text { (mm Hg) }\end{array}$} & Total \\
\cline { 2 - 5 } & $<\mathbf{3 1}$ & $\mathbf{3 1 - 3 8}$ & $\mathbf{3 3 8}$ & \\
\hline $1001-1499$ & 9 & 0 & 0 & 9 \\
\hline $1500-2499$ & 6 & 56 & 0 & 62 \\
\hline$\geq 2500$ & 0 & 2 & 91 & 93 \\
\hline TOTAL & $\mathbf{1 5}$ & $\mathbf{5 7}$ & $\mathbf{9 2}$ & $\mathbf{1 6 4}$ \\
\hline
\end{tabular}

Table 3: Mean BP (MBP) and Birth Weight

\begin{tabular}{|c|c|c|c|c|}
\hline \multirow{2}{*}{$\begin{array}{c}\text { Birth weight } \\
\text { (Grams) }\end{array}$} & \multicolumn{3}{|c|}{$\begin{array}{c}\text { No; of new borns with } \\
\text { MBP (mm Hg) }\end{array}$} & Total \\
\cline { 2 - 5 } & $<\mathbf{4 2 . 3}$ & $\mathbf{4 2 . 3 - 5 0 . 2}$ & $\mathbf{> 5 0 . 2}$ & \\
\hline $1001-1499$ & 8 & 1 & 0 & 9 \\
\hline $1500-2499$ & 2 & 58 & 2 & 62 \\
\hline$\geq 2500$ & 0 & 1 & 92 & 93 \\
\hline TOTAL & $\mathbf{1 0}$ & $\mathbf{6 1}$ & $\mathbf{9 3}$ & $\mathbf{1 6 4}$ \\
\hline
\end{tabular}

Table 4: Various studies on Blood Pressure estimation of Neonates

\begin{tabular}{|c|c|c|c|c|c|}
\hline Authors & Year & $\begin{array}{l}\text { Sample } \\
\text { size }\end{array}$ & $\begin{array}{c}\text { Gestational age of } \\
\text { neonates }\end{array}$ & $\begin{array}{c}\text { Ventilation/Inotropic } \\
\text { support }\end{array}$ & Method \\
\hline Zubrow et al ${ }^{14}$ & 1995 & 206 & $<37$ & + & Oscillometric \\
\hline Nwankuo MU et al ${ }^{11}$ & 1997 & 64 & $26-37$ & _- & Oscillometric \\
\hline Tin et $\mathrm{al}^{12}$ & 1999 & 400 & $<32$ & + & Doppler USG \\
\hline Vilarim et $\mathrm{al}^{15}$ & 2000 & 634 & $>37$ & _- & Oscillometric \\
\hline Kent et $\mathrm{al}^{13}$ & 2007 & 406 & $>37$ & - & Oscillometric \\
\hline Kent et $\mathrm{al}^{6}$ & 2009 & 147 & $28-36$ & _ & Oscillometric \\
\hline Present study & 2012 & 165 & $33-41$ & _ & Oscillometric \\
\hline
\end{tabular}

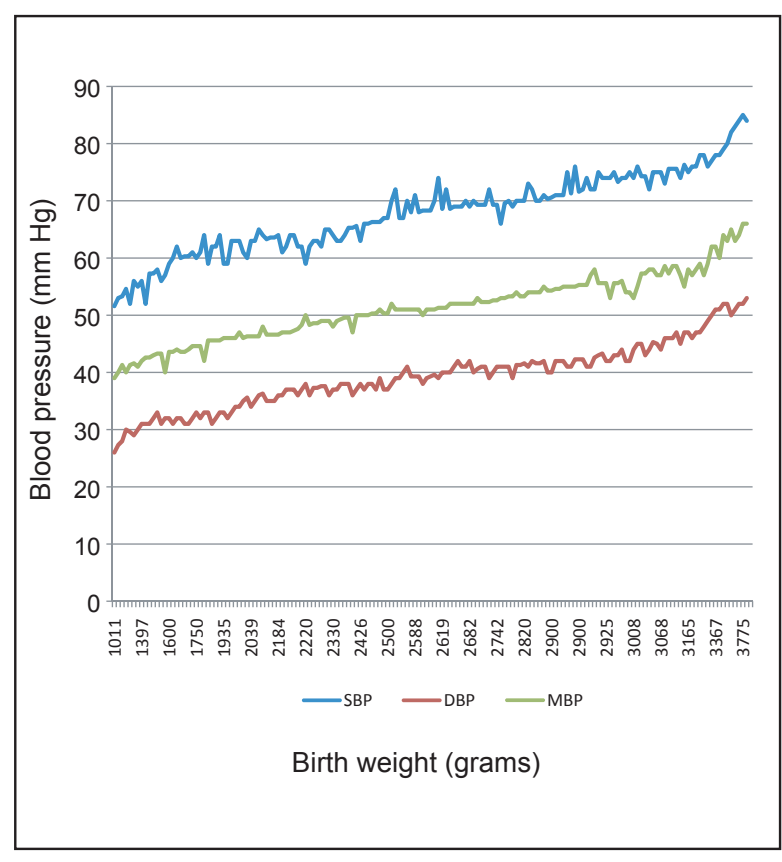

Fig 1: Systolic, mean and diastolic blood pressure values in relation to increasing birth weight

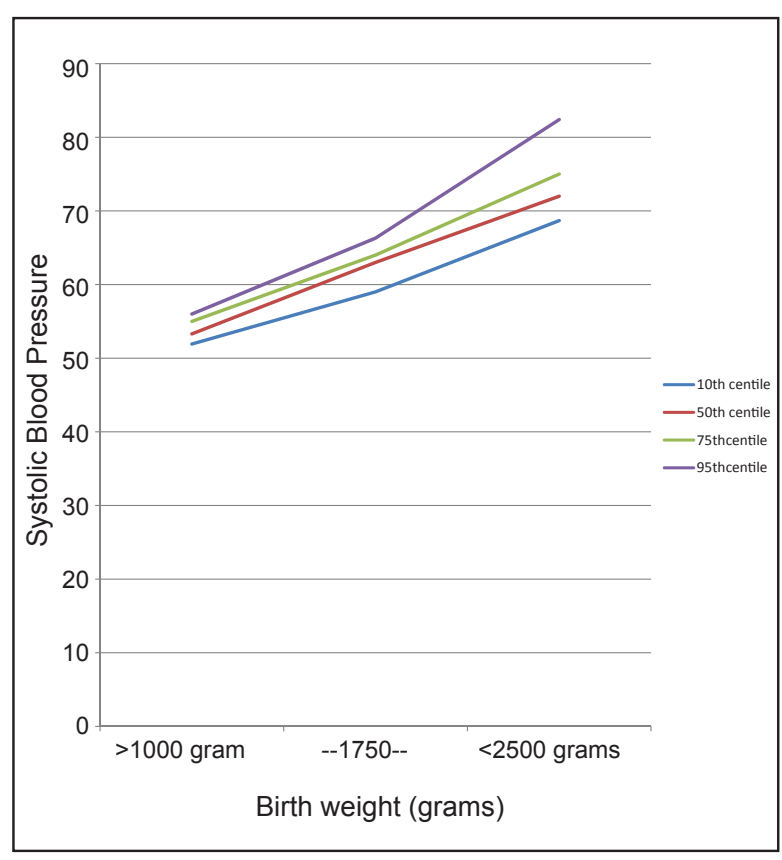

Fig 2: Centiles for Systolic Blood Pressure on Day 1 of Life for Birth Weight $\geq 1000$ grams 


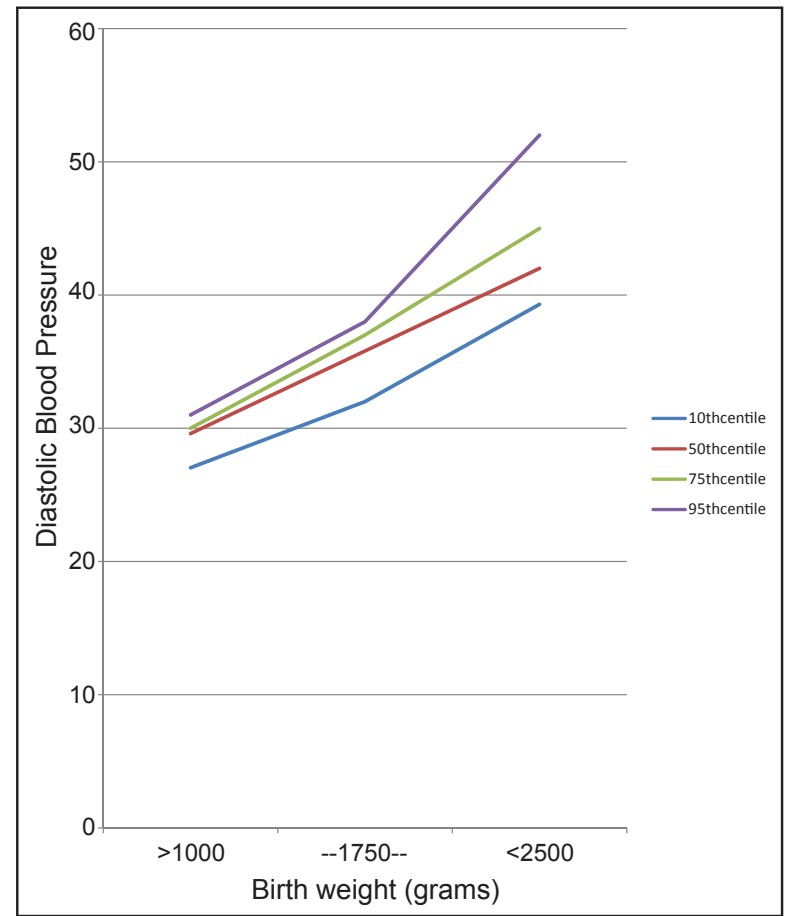

Fig 3: Centiles for Diastolic Blood Pressure on Day 1 of life for Birth Weight $\geq 1000$ grams

\section{Discussion}

This study provides normative non invasive Blood Pressure (NIBP) data - systolic, diastolic and mean BP values for 164 healthy newborns on day 1 of life, with gestation age 33-41 weeks ( mean $37 \pm$ 2 weeks) \& birth weight 1011 - 3820 grams (mean $2505 \pm 585$ ) in newborns aged $6-24$ hours, using oscillometric method. All infants were clinically and hemodynamically stable on day 1 of life.

The sample size is comparable to that of various published studies on BP in term and preterm newborns. Closest to our study in terms of sample size was study by Alison L. Kent et. al. ${ }^{6}$.

Hegyi et al provided a large dataset on BP in 1105 premature infants with birth weight ranging from 501 - 2000 grams ; however the study group consisted of heterogenous group of newborns including those on ventilator and /or on inotropic support ${ }^{7}$.

Oscillometric method was used in our study for BP assessment which is the now commonly employed method in clinical practice. Though, Invasive blood pressure monitoring is the gold standard, but besides clinical risks, it has other problems too. The pressure reading is affected by the mechanical properties of the intra-arterial catheter, the transducer system and presence of air bubbles ${ }^{8,9}$. These cause excessive

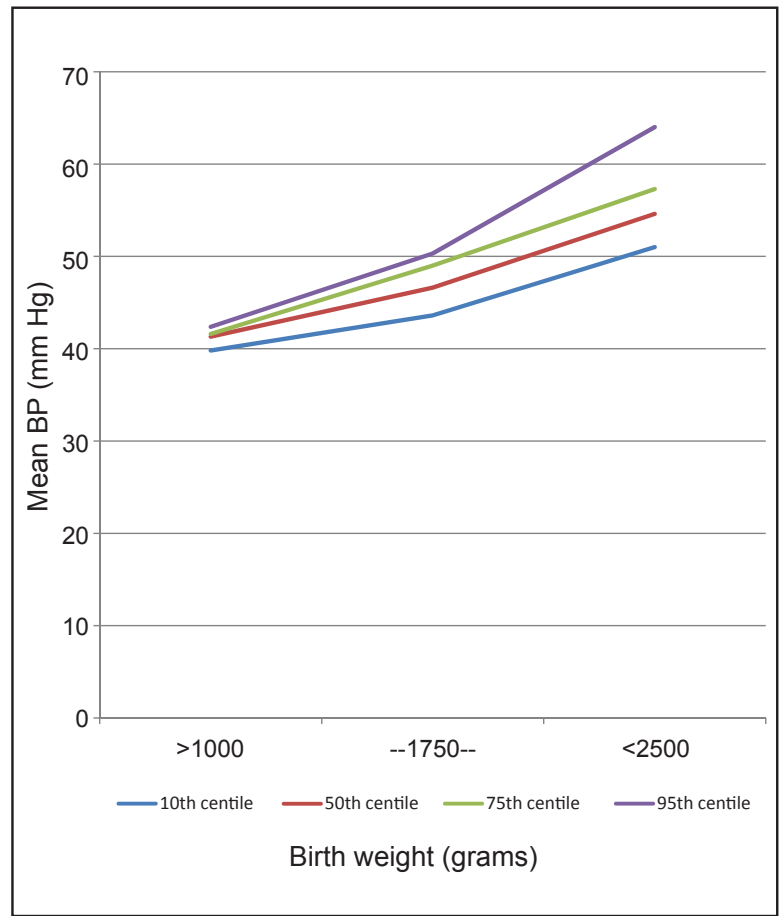

Fig 4: Centiles for Mean Blood Pressure on Day 1 of Life for Birth Weight $\geq 1000$ grams

damping leading to low systolic and high diastolic readings with minimal effects on mean $B P$. Therefore, while measuring BP by an arterial line, mean BP is assessed; but the normograms that are available for newborns are of systolic BP and hypotension and hypertension are also defined on basis of systolic $\mathrm{BP}^{10}$. Invasive methods thus fall into disadvantage. Moreover, catheterisation cannot be justified to obtain normative data in healthy newborns. Alison L.Kent et. al. ${ }^{6}$ and Nwankuo et. al. ${ }^{11}$ also used oscillometric method to determine normative BP values in babies.

The method used in our study emphasized the presence of restful state while taking the BP\& readings were taken after $6 \mathrm{hrs}$ of life $\mathrm{e}^{10}$. Alison L. Kent et al recorded BP on day 1 of life between 12 and 24 hours of life to allow for resolution of fetal circulation. Similar to our study, values were discarded if there was considerable movement of the infant and averages of three systolic, mean and diastolic were used for the analyses ${ }^{6}$.

In our study the average values of systolic, diastolic and mean BPs were $(67.9 \pm 7),(39.4 \pm 5.7)$ and $(51.4 \pm$ 5.9) $\mathrm{mm} \mathrm{Hg}$ respectively. In the study by Nwankwo $\mathrm{MU}$ et. al. ${ }^{11}$, the average systolic BP (in $\mathrm{mm} \mathrm{Hg}$ ) was $(65.2 \pm$ $1)$, diastolic BP $(36.1 \pm 0.8)$ and mean BP was ( $47 \pm 0.8)$. The median BP values were reported by Alison $L$ Kent et al in term babies as $65 \mathrm{~mm} \mathrm{Hg}, 45 \mathrm{~mm} \mathrm{Hg}$ and 48 
$\mathrm{mm} \mathrm{Hg}$ for systolic, diastolic and mean blood pressure respectively ${ }^{6}$.

In our study, a positive correlation was observed between BP \& birth weight. Tin et al also found that systolic blood pressure from $4-24$ hours of life was higher in babies with a higher birth weight ${ }^{12}$.

It was observed that the mean values of systolic, diastolic and mean blood pressure were higher for newborns with higher birth weight. These results were comparable to studies in the past by Alison L Kent et. al. ${ }^{6,13}$. Their study revealed that there was significant difference between the 28-29 week and 30-31 week group in terms of systolic BP and diastolic BP, with the younger gestation age group tending to have lower average BPs.

However, the results of our study were in contrast to that of Hegyi et al which concluded that birth weight and gestation age correlated with blood pressure limits only in infants with low Apgar score. In their study, in healthy infants, limits of systolic and diastolic blood pressure were independent of birth weight and gestational age ${ }^{7}$. Table 4 gives Sample size and method of BP estimation used in other studies and present study.

Limitations of the study: Small sample size and blood pressure values being single point values i.e. at first day of life only. Very small number of babies in very low birth weight category puts a difficulty for extension of study results to larger population.

Strength of the study: Despite its limitations, our study has biggest strength of being a pioneer study of its type in Indian literature. In India, where we face lot of neonatal morbidity and mortality, there is a need for improving our management protocols for sick neonates. While technology is improving day by day, we still need to improvise our knowledge of those parameters, which can be of paramount importance, but still not established. Oscillometric method of BP estimation is a safe, and most importantly cost effective such parameter. This study throws some light on the still dark issue of non-availability of BP values in Indian neonates.

\section{Conclusion}

Blood pressure on day 1 of life appears to be directly related to birth weight. The effect is seen in all (i.e. systolic, diastolic and mean) blood pressure values. This may be related to adaptive changes in cardiovascular system with increasing body size.
Recommendations: we recommend that more studies (of large sample sizes) should be carried out so that database of NIBP in Indian newborns can be created for both normal and low birth weight babies. Further, the studies should be carried out for the entire newborn period so that age wise normograms can be created.

\section{Acknowledgements: Nil \\ Funding: None \\ Conflict of Interest: None \\ Permission from IRB: Yes}

\section{References}

1. Lee KG. Identifying the high risk newborn. In: John P. Cloherty, Eric C. Eichenwald, Ann R.Stark (editors). Manual of neonatal care. 6th ed. Philadelphia: Lippincott Williams and Wilkins; 2010:42-3

2. Volpe JJ. Cerebral blood flow in newborn infant. J Pediatr 1979;130A:1125

3. Kim MS, Herrin JT. Renal conditions. In: John P.Cloherty, Eric C. Eichenwald, Ann R.Stark (editors). Manual of neonatal care. 6th ed. Philadelphia: Lippincott Williams and Wilkins; 2010:599-600

4. Ringer SA, James E. Common neonatal Procedures. In: John P.Cloherty, Eric C. Eichenwald, Ann R Stark (editors). Manual of neonatal care. 6th ed. Philadelphia: Lippincott Williams and Wilkins; 2010:659-60

5. Stebor AD, Short MA. Noninvasive BP measurement. Adv Neonatal Care 2005;5:252-61.

6. Kent AL, Meskell S, Falk MC, Shabdolt B. Normative blood pressure data in non-ventilated premature neonates from 28-36 weeks gestation. Pediatric Nephrol 2009;24:141-6

7. Hegyi T, Carbone MT, Anwar M, Ostfeld B, Hiatt M, Koons A, Martin JP et al. Blood pressure ranges in premature infants.I.The first hours of life. J Pediatr 1994;124:627-33

8. Weindling AM. Blood pressure monitoring in the newborn.Arch Dis Child 1989;64:444-7.

9. Nuntnarumit P, Yang W, Bada- Ellezey HS. Blood pressure measurements in the newborn. Clin Perinatol 1999;26:981-96.

10. Stork EK, Carlo W1, Kliegman RM, Fanaroff AA. Hypertension redefined for critically ill neonates. Pediatr Res 1984;18:321A.

11. Nwanko MU, Lorenz JM, Gardiner JC. A standard protocol for blood pressure measurement in the newborn. Pediatrics1997;99:E10. 
12. W. Tin. Systolic blood pressure in babies less than 32 weeks gestation in the first year of life. Arch Dis Child Fetal Neonatal ed 1999;80:F38-F42.

13. Kent AL, Kecskes Z, Shadbolt B, Falk MC. Normative blood pressure data in the early neonatal period. Pediatr Nephrol 2007;22:1335-41.
14. Zubrow AB, Hulman S, Kushner H, Falkner B. Determinants of blood pressure in infants admitted to neonatal intensive care units: a prospective multicentre study. Philadelphia blood pressure study group. J Perinatol 1995;15:470-9.

15. Vilarim JV, Alves JGB. Systolic and diastolic blood pressure of healthy newborn infants. J Pediatr (Rio J) 2000;76:287-9. 